\title{
A baseline survey looking at adult patients who no longer require percutaneous endoscopic gastrostomy (PEG) following joint intervention by a dietitian and a speech and language therapist (SLT)
}

\author{
E. J. Jarvis and L. E. Rogers \\ Home Enteral Nutrition Team, Lewisham PCT, Unit 2, Burgess Business Park, Parkhouse Street, Camberwell SE5 7TJ, UK
}

The benefits of PEG feeding are well documented ${ }^{(1)}$ but there is an impact on quality of life ${ }^{(2)}$. PEG tube removal can therefore be seen as a positive clinical outcome. Enterally fed patients should be supported by a co-ordinated multi-disciplinary team ${ }^{(1)}$, although the frequency of input and by whom is not specified. A cost saving of $21 \%$ per patient can be made with appropriate management by a nutrition team ${ }^{(3)}$. Retrospectively we examined the number of patients who were referred for PEG tube removal in 2006, the majority of whom were jointly managed. The role of the dietitian was to optimise oral nutrition and hydration intake whilst weaning them from PEG feeding. The role of the SLT was to treat dysphagia and maximise the safety of oral intake.

The caseload for 2006 was 230 patients and thirty-six (15.6\%) were referred for tube removal, of which twenty-eight (12.2\%) had dysphagia. PEG were not removed for palliative reasons. The ratio of males to females was 2.3: 1 . The age range was $29-83$ years at time of tube removal with a median of 66.5 years. Neurological diagnoses accounted for $53 \%$, whilst $47 \%$ had cancer $(94 \%$ had head and neck cancer). The length of time PEG feeding was required ranged from 3 to 91 months with an average of 15.2 months. Of those patients who where enterally fed for longer than 12 months (30.5\%), the majority (82\%) had a neurological diagnosis.

The number of patients who had their PEG tube removed was considerable. This positive outcome was the direct result of joint working. One study ${ }^{(4)}$ concluded that a positive clinical outcome is unlikely for older patients ( $>65$ years) with diagnoses other than localised head and neck cancer. Our results contraindicate this, highlighting a median age of 66.5 years with a predominant neurological diagnosis in those patients who were referred for PEG removal. Another study ${ }^{(5)}$ showed only one tube removal from ninety-nine patients with neurological impairment with a mean follow-up time of 3 months. Our results support this, highlighting the need for long-term intervention from a dietitian and SLT, as $97 \%$ of our patients needed a PEG for considerably longer than 3 months.

Our current figures for 2007 show similar trends. This highlights the need for more research leading to the development of standards with regards to the long-term multi-disciplinary management of all patients with PEG. The regular management of this client group has resulted in $12.2 \%$ of the caseload being discharged in one year. This is advantageous with regard to positive clinical outcome and has potential financial benefits.

1. NICE (2006) Nutrition support in adults: Oral Supplements, Enteral Tube Feeding and Parenteral Nutrition. NICE August 2006. Available at: http:// www.nice.org.uk.

2. Brotherton A, Abbott J \& Aggett P (2006) J. Hum Nut. Diet. 19 (5), 355-67.

3. Scott F, Beech. R, Smedley F, Timmis L, Stokes E, Jones P, Roffe C \& Bowling TE (2005) Nutrition 21 (11-12), 1071-1077.

4. Naik AD, Abraham NS, Roche VM \& Concato J (2005) Aliment Pharmacol Ther 21 (9), 1155-1161.

5. Zalar AE, Guedon C, Piskorz EL, Sanchez Basso A \& Ducrotte P (2004) Acta Gastroenterol Latinoam 34 (3), 127-132. 